\title{
IOT based Facial Recognition Door Access Control Home Security System
}

\author{
Anjali Patel \\ Mtech SS \\ Gyan Ganga Institute of Technology and \\ Sciences, Jabalpur
}

\author{
Ashok Verma \\ Associate Professor \\ Gyan Ganga Institute of Technology and \\ Sciences, Jabalpur
}

\begin{abstract}
This paper deals with the idea of secure locking automation utilizing IOT for door unlocking system to provide essential security to our homes, bank lockers and related control operations and security caution through the GSM module .It uses an image capturing technique in an embedded system based on raspberry pi server system. RPi (Raspberry pi) controls the video camera for catching it for turning on a relay for door unlocking. The module contains a secured face recognizer for automatic door unlocking. The camera catches the facial picture and compares it with the image which is stored in the database. If the picture is found in the database then the door lock opens otherwise it will produce a SMS that an unknown person is trying to gain access.
\end{abstract}

\section{Keywords}

Facial Recognition; Internet of Things(IOT);Image matching; Sensor System; Digital camera; Raspberry Pi 3;Person Identification

\section{INTRODUCTION}

In these modern times, home security is the need of the hour for the development of society as a whole which in turn will help make our cities smart, so the concept of facial recognition to gain access of the house is an idea which is used to make our place of living more secure. A facial recognition system is a system which captures facial images and verifies the identity of a person using a digital camera.

The human face assumes an essential part in our social association, passing on individuals' character. Utilizing the human face as a key to security, biometric confront acknowledgment innovation has gotten tremendous consideration in the previous quite a while because of its potential for a wide assortment of utilizations.

A facial acknowledgment framework is a framework which gets facial pictures and confirms the character of a man using a propelled camera . It is an application fit for distinguishing or checking a man from a computerized picture. One approach to do this is by looking at chose facial components from the picture and a face database.

As stood out from other diverse biometrics frameworks utilizing unique mark/palm print and iris, confront acknowledgment has unmistakable favorable circumstances due to its non-contact handle. Face pictures can be caught from a separation without touching the individual being recognized, and the ID does not require participating with the individual.

It is normally utilized as a part of security frameworks and can be contrasted with different biometrics. It has additionally turned out to be main stream as a commercial recognizable proof and advertising instrument.

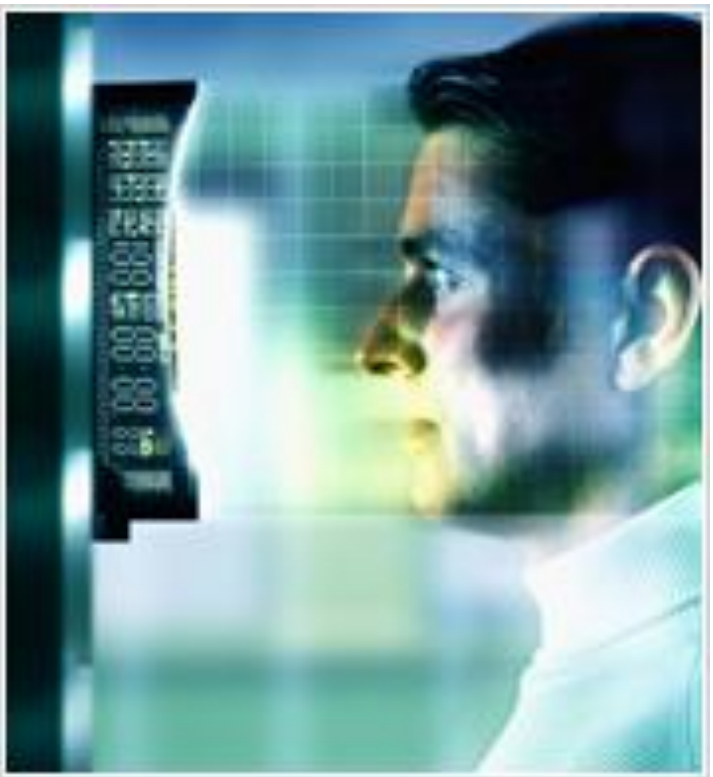

Fig 1: Facial Recognition

Internet of things: The internet of things, additionally called the internet of articles, refers to a remote system between items. The term IOT has come to portray various advances and research teach that empower the web to connect into this present reality of physical articles.

There are various technologies that enable IOT:

- $\quad$ RFID and near-field communication

- Optical tags and quick response codes

- Bluetooth low energy

- ZigBee

In this work we utilized raspberry pi 3 is a single board PC created in the United Kingdom by the raspberry pi establishment. Raspberry pi has many generations. What we are using here is pi3.It replaced pi2 model b in February 2016. Raspberry pi3 is believed to be approximately $80 \%$ faster than $\mathrm{RPi} 2$ in parallelized task. . Price is in between US $20 \$$ to $35 \$$. It has architecture of ARM v8(64/32 bit), Broadcom BCM 2837 System on chip used along with the CPU of $1.2 \mathrm{GHz}$ $64 / 32$ bit quad core ARM cortex A53. The memory of Raspberry pi 3 is $1 \mathrm{~GB}$ and the storage is in the micro SDHC slot. It has an additional feature of Wi-Fi and Bluetooth as compared to other versions of Raspberry Pi. 


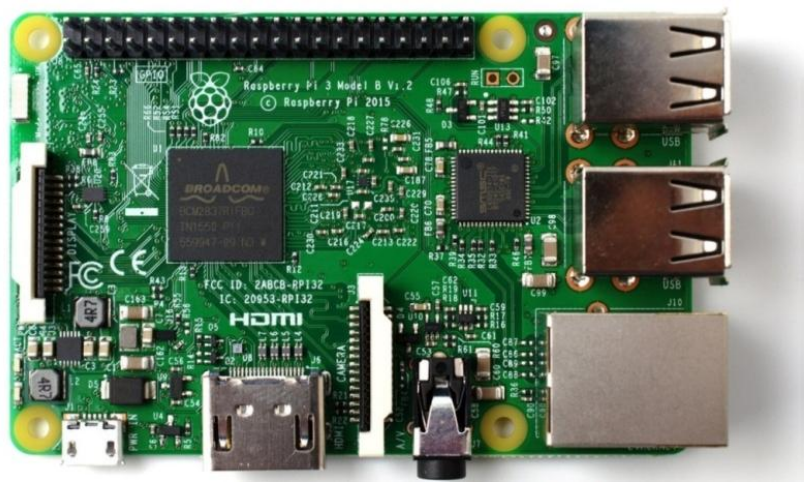

Fig 2: Raspberry Pi 3

IBM Blue mix is an open standard cloud based platform as a service (PaaS) developed by IBM. It supports several programming languages and services as well as integrated DevOps to build, run, deploy and manage all types of applications on the cloud. Bluemix depends on Cloud Foundry open technology and keeps running on Soft Layer infrastructure. Bluemix supports several programming languages including Java, Node.js, PHP, Swift, Python, Ruby, etc.

IBM Watson IOT: It is a cognitive system that learns from, and infuses intelligence into a physical world.

Node-Red is a programming device for wiring together hardware gadgets, APIs, and online organizations in new and intriguing ways. It gives a program-based supervisor that makes it easy to wire together streams utilizing the extensive variety of hubs in the palette that can be passed on to its runtime in a single click.

The light-weight runtime depends on Node.js, taking full perspective of its, occasion driven, on-blocking model. This makes it immaculate to continue running at the edge of the system on ease equipment, for example, the Raspberry Pi and also in the cloud.

In the late time, cloud computing has advanced as a net driven, benefit arranged processing model. As characterized by National Foundation of Measures and Innovation (NIST), cloud computing is show which empowers the advantageous, on-request organize access to a common pool of configurable registering assets (e.g., servers, administrations , applications, systems, stockpiling, and systems,).

In a facial recognition security system the Raspberry pi is combined with Cloud Computing to provide the communication between the person and the security system. Cloud is a service provider or a type of internet based computing that provides shared computers processing resource and other devices on demand.

Cloud computing is a gathering of advances that permit IT assets to be virtualized, utilized on an on-request premise and conveyed by means of the Web as administrations. Cloud computing can be viewed as another processing worldview in so far as it permits the use of a figuring framework at least one levels of deliberation, as an on-request benefit made accessible over the Web or other PC organize. It is sold on request, normally incrementally or the hour; it is versatile - a client can have to such an extent or as meagre of an administration as they need at any given time and the administration is completely overseen by the supplier. Due to its elements of more prominent adaptability and accessibility at lower cost, Cloud computing is a subject that has been getting a decent arrangement of consideration.

Cloud computing can be ordered into 4 sorts on the premise of area where the cloud is facilitated:-

- Public Cloud: An open cloud is one in which the framework and other computational assets that it contains are made accessible to the overall population over the Web. It is possessed by a cloud supplier offering cloud administrations and, by definition, is outside to an association.

- Private Cloud: A private cloud a restrictive system or a server farm that provisions facilitated administrations to a predetermined number of individuals. It might be overseen either by the association or an outsider, and might be facilitated inside the association's server farm or outside of it.

- Community Cloud: A people group cloud is fairly like a private cloud; however the foundation and computational assets are shared by a few associations that have regular protection, security, and administrative contemplations, instead of for the restrictive utilization of a solitary association.

- Hybrid Cloud: A cross breed cloud is a piece of at least two mists (private, group, or open) that stay extraordinary elements yet are bound together by institutionalized or restrictive innovation that empowers interoperability.

Cloud computing uses three conveyance models by which diverse sorts of administrations are conveyed to the end client. The three conveyance models are the SaaS, PaaS and IaaS which give framework assets, application stage and programming as administrations to the purchaser.

- Software-as-a-Service - SAAS is characterized as a product appropriation display in which applications are facilitated by a seller or specialist co-op and made accessible to clients over a system. Otherwise called "on request" programming, it is the most develop kind of Cloud computing on account of its high adaptability, demonstrated bolster administrations, improved versatility, lessened client support, and diminished cost due to their multi-principle designs. It is a model of programming arrangement whereby at least one application and the computational assets to run them are given to use on request. Its primary intention is to decrease the aggregate cost of equipment and programming advancement, upkeep, and operations. Security arrangements are completed predominantly by the cloud supplier. The cloud supporter does not oversee or control the hidden cloud framework or individual applications, with the exception of inclination choices and constrained regulatory application settings.

- Platform-as-a-Service - PAAS gives foundation on which programming engineers can fabricate new applications or amplify existing applications without requiring the need to (buy advancement, QA, or generation server framework. It is a model of programming arrangement where the figuring stage is given as an on-request benefit whereupon applications can be created and sent. Its primary reason for existing is to lessen the cost and unpredictability of purchasing, loading, and dealing with the fundamental equipment and programming segments of the stage, including any required program and database advancement instruments. The cloud supporter has control over applications and application environment settings of the stage. Security 
arrangements are part between the cloud supplier and the cloud endorser.

- Infrastructure-as-a-Service - Infrastructure-as-aService (IaaS) is a model of programming organization whereby the fundamental figuring foundation of servers, programming, and system gear is given as an on-request benefit whereupon a stage to create and execute applications can be built up. Its fundamental reason for existing is to abstain from buying, lodging, and dealing with the essential equipment and programming framework segments, and rather get those assets as virtualized items controllable by means of an administration interface. The cloud supporter for the most part has expansive flexibility to pick the working framework and improvement environment to be facilitated Security arrangements past the essential foundation are done predominantly by the cloud endorser.

The cloud stage is utilized as a part of this venture is IBM Blue mix. It is an open standard, cloud based stage for building, overseeing and running utilizations of different types (web, versatile, huge information, and new brilliant gadgets, so on).

The designer utilizing Blue mix for:

- To quickly put up items and administrations for sale to the public at lower cost.

\section{LITERATURE REVIEW}

In today's fast paced and ever changing world security is one of the basic needs of our lives. Use of technology in the field of security plays an important role in increasing the security as well as reducing the manpower efforts.

Y. Januzaj. et al. [1] proposed real time access control for face recognition using, Raspberry pi instead of GSM services and relay. The limitation of the work was it couldn't control the background light situation and ambient light conditions.

H.Lwin.et al.[2] has proposed a door lock access system which consists of three subsystems: to be specific face recognition ,face detection, and automated door access control. Face recognition is actualized by using the PCA (Principal Component Analysis ). The door will open itself for the known person in command of the microcontroller and caution will ring for the unknown person. Demerit of this system is input images are taken through a web camera continuously until the 'stop camera' button is pressed. Somebody is required at the location to check unauthorized person's images or status of the system and take further appropriate action. Personal computer (PC) is associated with the microcontroller, The entire system will not work if $\mathrm{PC}$ is crashed or Non-Function..

M. Chowdhury.et al. [3] had implemented security system where if any person came at the door it was notified to the home owner via e-mail and twitter then the user could see the person standing at the door using camera from remote location. The image of the person got captured and sent to twitter and e-mail. They stated that user couldn't control the door remotely. They had concluded that this system was useful for preventing unauthorized access. The limitation of this work was that the alert generated was sent to the mail and twitter account but if the user didn't have internet connection on his/her phone, he/she couldn't check the mail and couldn't recognise that any unauthorized person was trying to access the door.

G.senthilkumar.et.al. [4] proposed a work on Embedded Image Capturing System Using Raspberry Pi. In this work, they captured the image and compared it with the database but the limitation was the system couldn't work properly in the ambient light condition.

M. Carikci et al. [5] proposed a work on A Face Recognition System based on Eigen face method in which they used Eigen method for face recognition and Euclidean distance method to compare the image of the person concerned with the images in the database. It was very efficient and fast method and also gave high accuracy.

S. Jogdand.et.al [6] proposed a work on Implementation of Automated Door Accessing System with Face Design and Recognition in which they used Viola Jones method for face detection and PCA (Principal Component Analysis) for the comparison of images. The limitation of this work was that it is not robust and the efficiency is less.

U. Sowmiya.et al [7]. Developed to connect any door with internet. In this system user also implemented PIR sensor and camera. PIR sensor used for detecting person and camera used for capturing the video of the person who comes at the door. The video was sent through $3 \mathrm{~g}$ dongle to authorized person. They had also discussed some advantages of this system. They had concluded use of this system in banks, hospitals etc. But their proposed model didn't provide the facility of sending messages to the authorized people.

J. Kartik et al [8]. Have proposed two systems are proposed, one is based on GSM technology and other uses a web camera to detect the intruder. The first security system uses a web camera, installed in house premises, which is operated by software installed on the PC and it uses the Internet for communication. The camera identifies movement of any intruder before the camera measurements or camera range. The product imparts to the planned client through Internet arrange and in the meantime, it gives a sound alarm. The second security system is SMS based and utilizes GSM innovation to send the SMS to the owner.

\section{METHODOLOGY AND WORKING OF THE PRPOPOSED SYSTEM}

The proposed works are as follows:

- Interfacing of camera to capture live face images.

- Create a database of authorized person if they exist.

- Capturing current image, save it and compare with the database image.

- Interface GSM module to send alert to authorized person while unlocking the locked door in the form of SMS and CALL.

- The project can also be used for surveillance. For instance, it can capture the images of unidentified individuals and store it which can later be used to determine the impostors who tried to gain illegitimate access.

- Interface relay as an output. 


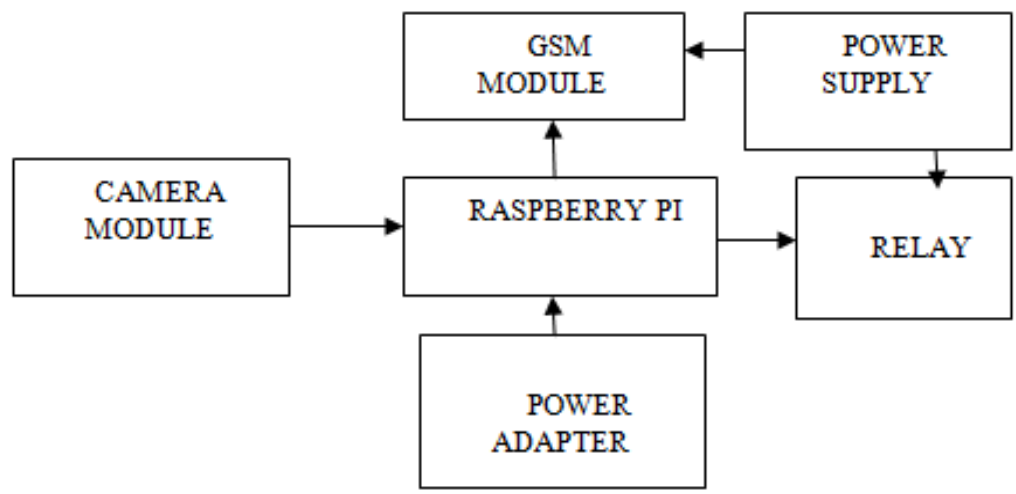

Fig 3: Block diagram of "Raspberry pi based face recognition system for door unlocking".

The system will works in two different parts. The first part is for capturing and creating a database by storing the image. And the second one is to compare the image with the stored images in the database .For feature extraction we will use Eigen faces methodology and Euclidian distances will used for recognition of the face.

Camera module: Camera module is pi camera interfacing to the raspberry pi module. It is used to capture images and send the clicked images to the raspberry pi module. Camera contains LEDs and flashes to handle that light condition that is not explicitly supplied by the environment and these light conditions are known as ambient light conditions.

Raspberry pi module: raspberry pi 3 module is a small computer board. When an image is taken by raspberry pi it is

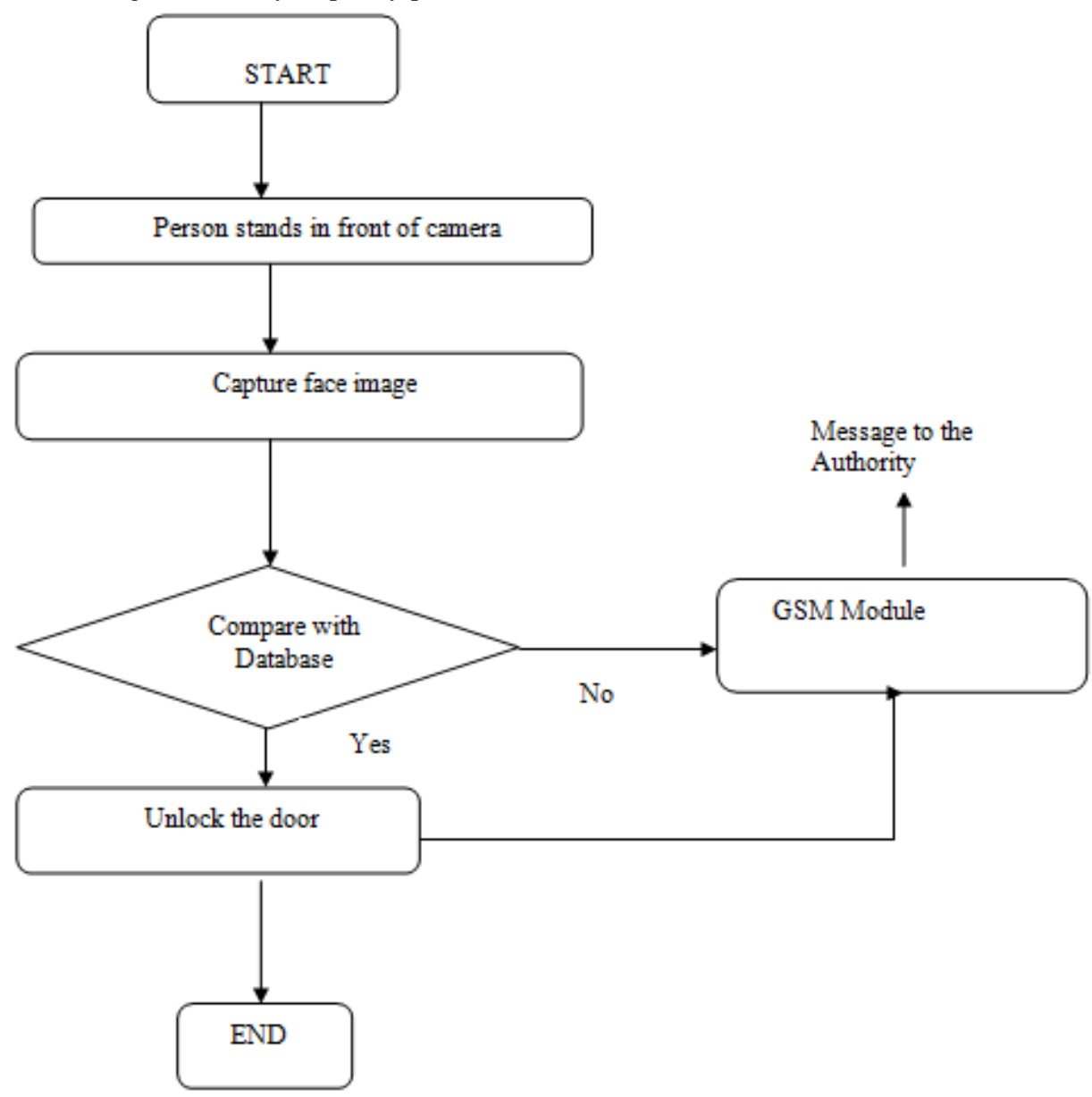

Fig 4: Flowchart of Image capturing and database comparison compared with database image. For the first time when we capture an image to Create a database raspberry pi module captures many images to create a database in the system and this database is compared with the live captured images. After comparing the two images, based on whether the output is positive or negative it gives commands to GSM module.

GSM Module: GSM module is used to send a message to the authorized people based on the output. If the output is positive "Information matched Access granted" message will be sent to the authorized people, otherwise in case of unauthorized access it will send an "Access denied. Some unknown person is trying to unlock the door". Message to the certified users of the system. 


\section{RESULT ANALYSIS}

The result in the creation of real time database are recorded. The real time database is created by using python. While executing it produces 30 images of each subject. . Likewise, databases should be created for at least 10 individuals and it creates each image size of about $100 * 130$ pixels of height and width..

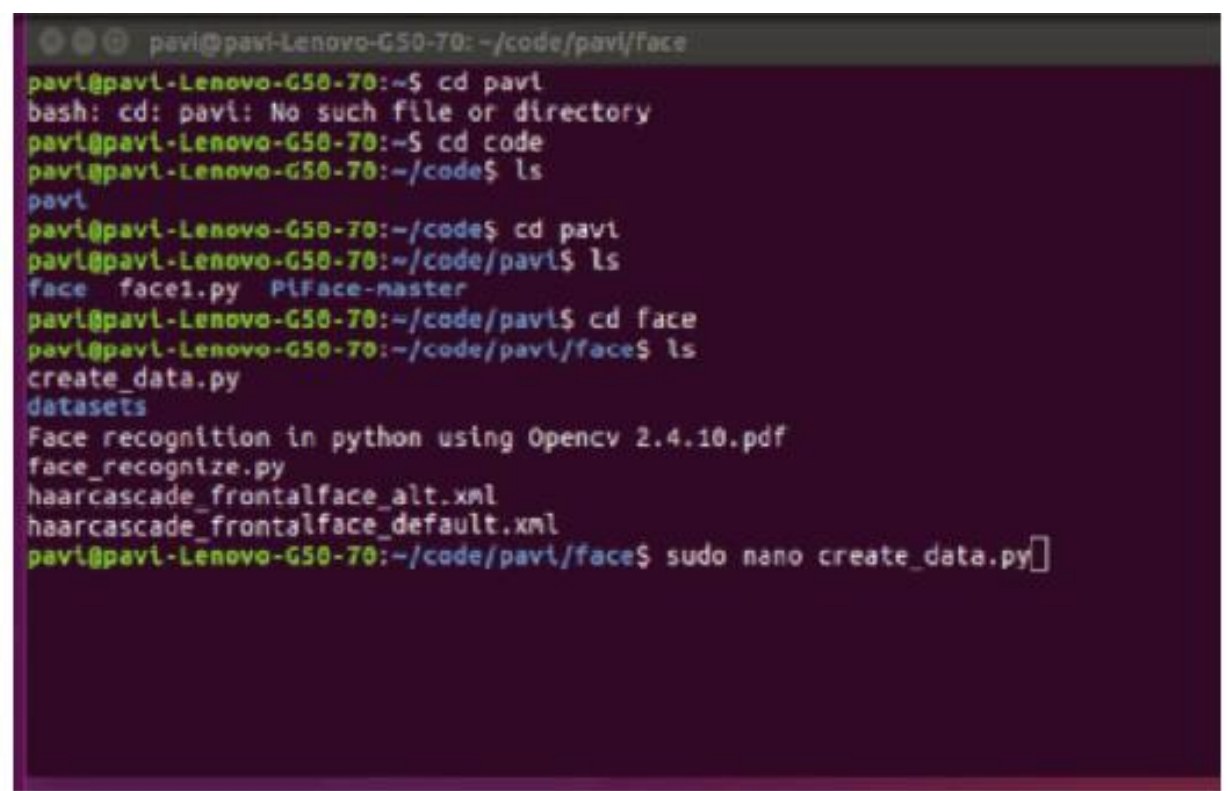

\section{Fig 5: Creation of Real Time database}

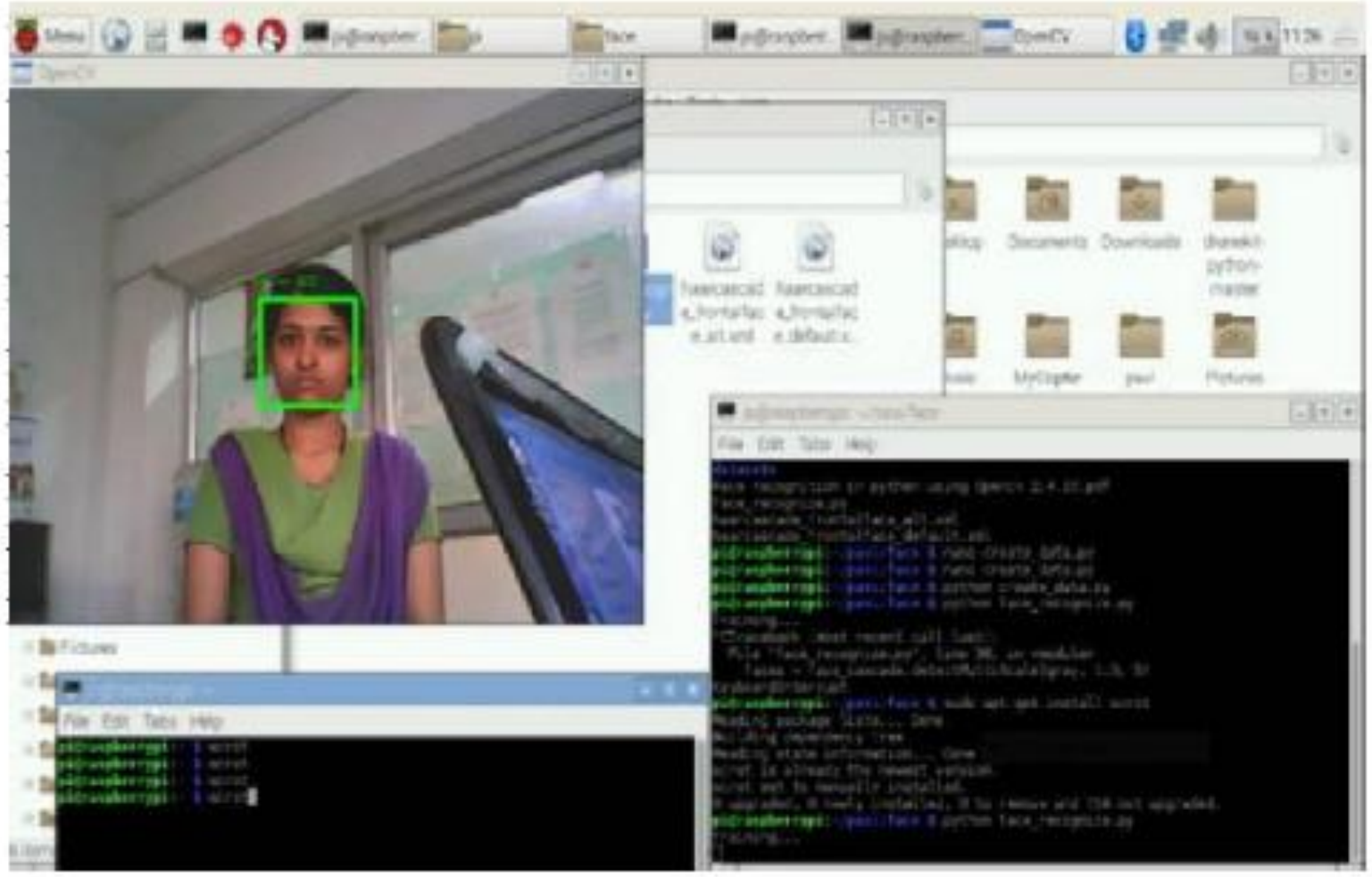

Fig 6: Facial recognition using raspberry pi 


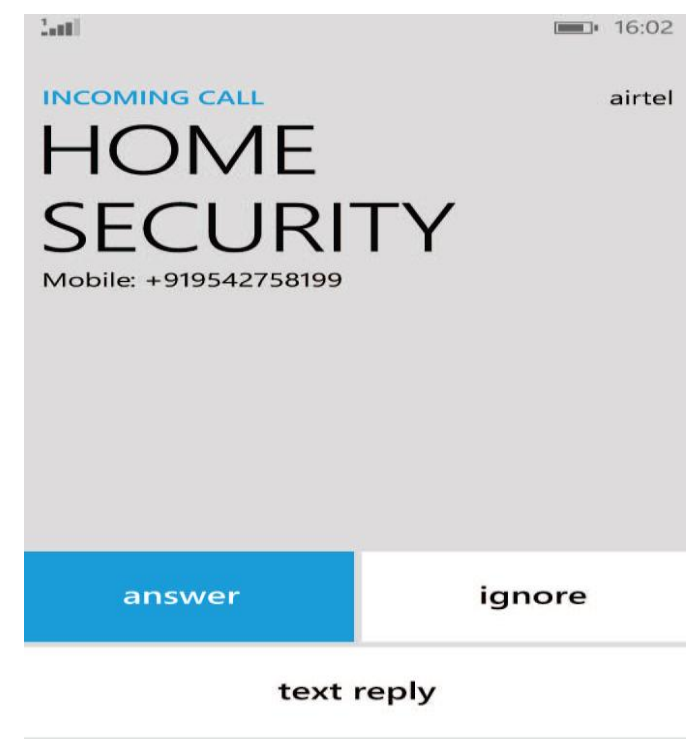

Fig 7: Call From GSM module

Table 1. Accuracy of Proposed System

\begin{tabular}{|c|c|}
\hline Simulating parameter & Range \\
\hline No of training images & 100 images \\
\hline No of testing images & 10 images \\
\hline Size of image & $100 * 130$ \\
\hline Normal condition & $95 \%$ \\
\hline Illumination condition & $83 \%$ \\
\hline
\end{tabular}

S15

Figure 8: Storing database of each individual in specific directory 


\section{CONCLUSION}

The arrangement of a facial recognition system using raspberry pi can make the system littler, lighter and work successfully utilizing lower control use, so it is more convenient than the pc- based face recognition system. It is open source software on Linux. Also, send a security alert message to the authorized person utilities. We are also providing power backup for the smooth and continuous functioning of the system in case of power failure. The power bank is used to charge the Raspberry Pi so there is less chance to slow down the system.

This development scheme is cheap, fast, and highly reliable and Raspberry pi takes less power and provides enough flexibility to suit the requirement of different people.

\subsection{Future work}

- If a blacklisted person tries to open the door, the system will send a message to the admin using GSM module regarding the same.

- A real time speaking assistant can be deployed to make the system more user -friendly and efficient.

- Highly secure protocols such as TLS can be deployed to ensure there is no security breach.

\section{REFERENCES}

[1] Januzaj, Y., Luna, A., Ramaj, V. 2015 Real time access control based on Facial Recognition.

[2] Lwin, H., Khaing, A., Tun, H. 2015.Automic door access system using face recognition.
[3] Chowdhury, M., Nooman, S. 2013. Access Control of Door and Home Security by Raspberry Pi through Internet.

[4] Senthikumar, G., Gopalkrishnan, K., Sathish Kumar, V. 2014 Embedded Image Capturing System Using Raspberry Pi System.

[5] Çarıkçı, M., , Özen, F. 2012 A Face Recognition System Based on Eigen faces Method.

[6] Jogdand, S., Karanjkar, M. 2015Implementation of Automated Door Accessing System with Face Design and Recognition.

[7] Sowmiya, U., shafiq mansoor, J. 2015 Raspberry pi based home door security through $3 \mathrm{~g}$ dongle.

[8] Kartik J. Srimadhavan V. 2013 SMS Alert and Embedded Network Video Monitoring Terminal.

[9] Sahani, M., Nanda, C., Sahu, A., Pattnaik, B. 2015Web Based Online Embedded Door Access Control and Home Security System Based on Face Recognition,.

[10] Mulla,,M., Patil, R. 2015 .Facial Image Based Security System using PCA.

[11] Gubbi, Jayavardhana,. (2013) Internet of Things (IOT): A vision, architectural elements, and future directions. Future Generation Computer Systems 29.7: 1645-1660.

[12] Raspberry Pi Foundation http://www.raspberrypi.org/downloads/.
[Online] 